relatively short time horizons, there is a clear need for longer term studies to investigate resource use of ICU survivors. Our findings should help to inform the design and reporting of such studies.

\section{P52 PREDICTORS OF EMERGENCY DEPARTMENT ATTENDANCE RATES IN SMALL AREA POPULATIONS}

doi:10.1136/jech.2010.120477.52

${ }^{1} \mathrm{G}$ Rudge, ${ }^{1} \mathrm{~S}$ Fillingham, ${ }^{2} \mathrm{~K}$ Sidhu, ${ }^{1} \mathrm{M}$ Mohammed. ${ }^{1}$ School of Health and Population Sciences, The University of Birmingham, Birmingham, UK; ${ }^{2}$ West Midlands Strategic Health Authority, Birmingham, UK

Objectives Emergency Department (ED) use is known to be related to both socio-economic deprivation and distance to hospital. However, hospitals are often located in urban areas close to deprived populations. The objective of this study is to investigate the relationship between distance and attendance at EDs, net of deprivation. Method Multiple linear regression models were used to explore the relationship between deprivation and distance to hospital with agesex standardised ED attendance rates at neighbourhood level. Distance to a Minor Injury Unit (MIU) was also included. Interaction between distance and deprivation was investigated. Separate models for children and adults were used because some populations had access to a dedicated children's ED.

Setting The Government Office Region of the West Midlands, in central England (population 5.4 million).

Population Attendances of West Midlands residents to EDs in acute hospitals in financial year 2007/08 $(n=1465355)$ within 3482 Lower Level Super Output Areas (LSOA), each containing approximately 1600 residents.

Outcome Measures The response variable was the directly age-sex standardised ED attendance rate for each LSOA (square-root transformed). Predictor variables were distance from LSOA centroid to nearest ED and nearest MIU in kilometres and income deprivation score (high score=high deprivation).

Results There was a significant positive relationship between attendance and deprivation, $\beta$ coefficient $=0.96(p<0.001)$ in adults and $0.68(p<0.001)$ in children. There was a significant negative relationship between attendance and distance, the $\beta$ coefficient $=-0.24(p<0.001)$ in adults and $-0.37(p<0.001)$ in children. Child attendance appeared more sensitive to distance than adult attendance but less sensitive to deprivation. Attendance in deprived neighbourhoods was more sensitive to distance than in less deprived ones. For adults, at the third quartile of deprivation, $\sqrt{ }$ attendance rate $=-0.32$ per $\mathrm{km}+19.50$ compared to -0.18 per $\mathrm{km}+15.86$ at the fist quartile. For children, at the third quartile of deprivation, $\sqrt{ }$ attendance rate $=-0.49$ per $\mathrm{km}+21.30$ compared to -0.30 per $\mathrm{km}$ +17.95 at the first quartile. The paper goes on to describe the models in greater detail, (including the effect of MIUs).

Conclusion Attendance at EDs is sensitive to income deprivation at neighbourhood level but distance from hospital appears to modify this relationship differently in deprived areas than in affluent ones. Also, children's attendance appears to be more sensitive to distance than that of adults. This is useful to understand when comparing ED attendance between and within populations.

\section{P53 MORTALITY AFTER HOSPITAL ADMISSION CLEARLY VARIES BETWEEN ATHEROSCLEROTIC DISEASES LOCATED AT DIFFERENT VASCULAR BEDS}

doi:10.1136/jech.2010.120477.53

I Vaartjes, D E Grobbee, Bots. Julius Centre for Health Sciences and Primary Care, University Medical Centre Utrecht, Utrecht, The Netherlands

Objective Most studies providing data on survival in patients with atherosclerosis only address a single disease site: heart, brain or legs.
Therefore, our objective was to determine risk of death after first hospital admission for atherosclerotic disease located at different sites.

Design Hospital-based follow-up study.

Setting and Participants A nationwide cohort of patients hospitalised for the first time for acute myocardial infarction (AMI), peripheral arterial disease of the lower extremities (PAD) or ischaemic stroke was identified through linkage of national registers in 1995, 1997 and 2000. The total population of the Netherlands in 1995, 1997 and 2000 was 15424122 (men 7627 428, women 7 796640), 15567107 (men 7696803 , women 7870304 ) and 15863950 (men: 7846317 , women: 8017633$)$, respectively.

Main Outcome Measure Twenty-eight-day, 1-year and 5-year mortality rate in AMI patients was compared to mortality rate in ischaemic stroke patients and $\mathrm{PAD}$ patients by estimating RR (with $95 \% \mathrm{CI}$ ). Cox's proportional hazard models were used to adjust for age.

Results Case fatality was highest for ischaemic stroke patients though lowest for PAD patients. In patients 55 to 74 years, large differences in risk of death were observed between AMI patients and $\mathrm{PAD}$ patients (men: RR 9.62; 95\% CI 5.78 to 16.02, women: RR 14.28 ; $95 \%$ CI 6.68 to 30.52 ) In contrast, the 5 -year risk of death was similar for male AMI and PAD patients and differences in risks of death between AMI and ischaemic stroke patients largely attenuated.

Conclusions The dynamics of mortality over time clearly differ between atherosclerotic diseases. Short-term mortality is higher for cardiac and ischaemic stroke patients compared to PAD patients. However, the risk of death increases considerably over follow-up times for PAD patients, and 5 years after first hospital admission the differences in risks of death between AMI and PAD patients and between AMI and ischaemic stroke patients have largely attenuated.

\section{P54 MIGHT FINANCIAL CUTS DRIVE NHS DECISION-MAKING "DOWNSTREAM"? A OUALITATIVE STUDY OF FACTORS AFFECTING PUBLIC HEALTH DECISION-MAKING}

doi:10.1136/jech.2010.120477.54

L Orton, F Lloyd-Williams, D Taylor-Robinson, M O'Flaherty, S Capewell. Division of Public Health, School of Population Community and Behavioural Sciences, University of Liverpool, Liverpool, UK

Objectives To explore the process of public health decision-making, and the role of research evidence, taking cardiovascular disease (CVD) as a case study.

Design In-depth interview study.

Setting and Participants Over 30 public health policy-makers and planners in CVD, including: commissioners, public health consultants, data analysts, librarians and knowledge managers at Primary Care Trusts; public health academics; lead consultant cardiologists; local and national guideline developers; and third sector staff.

Methods In-depth semi-structured interviews were recorded and transcribed verbatim. Transcripts and field notes were analysed using the constant comparative method.

Findings Participants reported that previously there was relatively little change in health care investment year on year. Consequently, it was seldom necessary to seek research evidence. However, changes had occurred in recent times. National Health Service (NHS) cuts had led to a more systematic prioritisation process necessitating the explicit use of research evidence. There was a sense that decisionmakers must now take stock of what they were doing and ascertain if it was evidence-based. Unfortunately, these cuts had also removed much of the opportunity for creative thinking and for trying out new and unproven innovations. Despite most participants wishing 
to address "upstream" issues, a focus on short-term national targets appeared to have constrained the adoption of population-level prevention initiatives in favour of "downstream" service development approaches. Furthermore, participants anticipated increased rationing of health care in the near future. Reliable research evidence was considered essential to inform rationing decisions. However, most academic research was perceived to be aimed at clinicians. Guidelines, particularly those produced by the National Institute for Health and Clinical Excellence (NICE), as well as local data (such as hospital statistics), were currently the main sources of evidence used by policy-makers and planners. In order to facilitate future decision-making, participants requested that researchers provide unequivocal evidence on the best approaches to service delivery.

Conclusions Most policy-makers and planners rely on NICE guidance and local data rather than directly accessing research evidence. Furthermore, public health decision-making in the NHS is constrained by organisational rigidity imposed by historic budgets and short-term national targets. Recent NHS cuts have led to increased systematisation and an emphasis on the evidence base. However, these cuts have also led to a focus on downstream interventions. In future, more effective health care rationing may require additional research on models of service delivery.

\section{P55 THE IMPACT OF NEO-LIBERAL "POLITICAL ATTACK" ON HEALTH: THE CASE OF THE "SCOTTISH EFFECT"}

doi:10.1136/jech.2010.120477.55

${ }^{1} \mathrm{C}$ Collins, ${ }^{2} \mathrm{G}$ McCartney. ${ }^{1}$ University of the West of Scotland, Paisley, UK; ${ }^{2} \mathrm{NHS}$ Greater Glasgow and Clyde, Glasgow, UK

Objective There is a prevalent, but as yet untested, view in Scottish society that the "Scottish Effect" (the excess mortality in Scotland after taking account of deprivation) is linked to the neo-liberal "political attack" against the organised working class implemented by the post-1979 UK Conservative governments. The objective is to develop and begin to test such a "political attack hypothesis".

Design Development of a "political attack hypothesis" underpinned by social science literature on UK and Scotland, and by a theory of change connecting neo-liberal "political attack" and mortality. Identification of key respects in which the West of Scotland in particular would seem to have been more susceptible to negative impacts of "political attack" on key upstream determinants of mortality. Testing with relevant data.

Setting West of Scotland and comparison areas consisting of other UK and European de-industrialised areas.

Results The links between such neo-liberal "political attack" and adverse health outcomes are plausible and have been examined in other contexts, including the former USSR. Evidence from the Fraser Institute and Luxembourg Income Study indicates that a neo-liberal policy agenda was implemented in the UK to an extent not seen elsewhere in Europe. Authoritative historical accounts indicate that the West of Scotland became a particular target for the UK political attack planned by the Conservative Party prior to its election in 1979. They also suggest that Scotland was disproportionately vulnerable to its adverse effects. This is confirmed by data on industrial employment, on housing tenure and on voting trends (taken as an indication of an intensifying sense of disempowerment and loss of control within Scotland during the period in which the "Scottish Effect" is known to have emerged).

Conclusion There is some evidence to support a "political attack" hypothesis in relation to the Scottish Effect. The hypothesis offers an important avenue for further investigation. Similarly elaborated hypotheses may also have broader relevance to other contexts where a radical neo-liberal agenda has been implemented.

\section{Area effects \\ P56 DOES AREA REGENERATION IMPROVE RESIDENTS' HEALTH AND WELL-BEING?}

doi:10.1136/jech.2010.120477.56

D Archibald. The University of St-Andrews, St-Andrews, UK

Background Despite $£ 12$ billion of UK government investment in the last 20 years, the impact(s) of regeneration initiatives on health remain unclear. The literature shows that some regeneration initiatives appear to have had positive effects on health, whilst others have had no, or even a detrimental effect. This however may be down to difficulties in designing appropriate evaluation studies rather than the effects of regeneration per se. Evaluations in this field have often lacked rigour and have thus rarely been able to explore the dynamic nature of area change that often accompanies the regeneration process.

This poster reports on the progress of the quantitative phase of a mixed-methods $\mathrm{PhD}$ study that employs a mixed-methods design to investigate the little understood effects of area regeneration on health in Scotland.

Objective To address limitations seen in previous evaluations such as:

Use of small case studies to make assumptions about the successes and/or failings of regeneration programmes.

Inability to follow individuals through time so that individuals' changing circumstances can be more securely related to the regeneration processes that they experience.

Use of unsatisfactory cross-sectional approaches that compare population characteristics before and after regeneration processes, which ignores the fact that resident population may have changed substantially during the regeneration period.

Methods This phase of the research will conduct a quantitative secondary analysis of data from the Scottish Longitudinal Study to explore the health and socio-economic effects of area regeneration on people's health and well-being in Scotland. We will compare outcomes for individuals who experienced regeneration between 1991 and 2001 with individuals living in similarly deprived areas throughout the same period that did not.

Conclusion Area-based regeneration is seen in the UK as a strategy for tackling the poor health and health inequalities that many of the poorest members of society experience simply by virtue of their socio-economic status. Thus, unlike many past evaluations, this longitudinal study will allow us to determine how regeneration has influenced those who actually experienced it by following individuals through time. This will benefit the wider public by helping to improve the rigour of the (currently limited) evidence base, which in turn may assist in the development of more effective policy aimed at tackling socio-economic deprivation through area-based initiatives.

\section{P57 PERSISTENT REGIONAL VARIATION IN TREATMENT OF HYPERTENSION}

doi:10.1136/jech.2010.120477.57

${ }^{1} \mathrm{~J}$ Mindell, ${ }^{1} \mathrm{~N}$ Shelton, ${ }^{1} \mathrm{M}$ Roth, ${ }^{1} \mathrm{M}$ Chaudhury, ${ }^{2} \mathrm{E}$ Falaschetti. ${ }^{1} \mathrm{Health}$ and Social Surveys Research Group, Department Epidemiology \& Public Health, UCL, London, UK; ${ }^{2}$ Cardiac Unit, UCL Institute of Child Health, London, UK

Objective To investigate risk factors for treatment for hypertension in people with survey-defined hypertension in England.

Design Cross-sectional health examination surveys.

Setting Three, nationally representative random samples of the general population living in private households in England-the Health Survey for England (HSE) in 2005, 2006, and 2007.

Participants A new, nationally representative sample is selected each year. A random sample of the free-living general population (HSE 